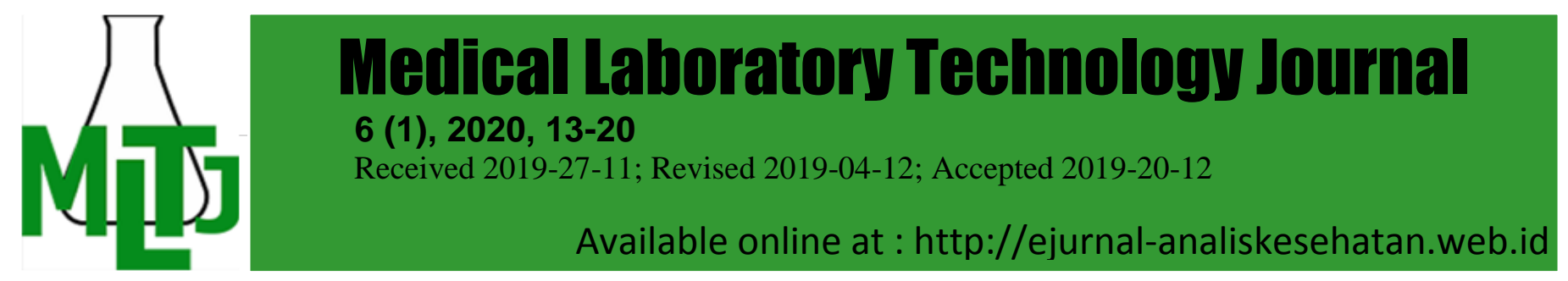

\title{
Animal Models with Metabolic Syndrome Markers Induced by High Fat Diet and Fructose
}

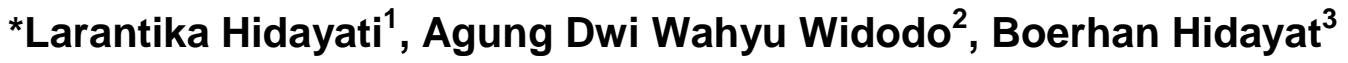

${ }^{1}$ Immunology Study Program, Postgraduate School of Airlangga University Jl. Airlangga No.4-6, Airlangga, Gubeng, Surabaya, East Java,60115, ²Department of Clinical Microbiology, Faculty of Medicine, Universitas Airlangga, Surabaya, Indonesia, ${ }^{3}$ Department of Pediatrics Faculty of Medicine, Universitas Airlangga, Surabaya, Indonesia. *Email: larantika19@gmail.com

DOI: 10.31964/mltj.v1i1.266

\begin{abstract}
Metabolic syndrome is lipid and non-lipid metabolism disorder due to the association of several factors such as physiological, clinical, biochemical, and interrelated factors. People with metabolic syndrome can be diagnosed by fulfilling 3 of 5 criteria, including obesity and increased waist circumference, increased TG levels, increased blood pressure, hyperglycemia, and decreased High-Density Lipoprotein (HDL) serum. The high-fat diet disrupts tissue lipid metabolism, so insulin resistance occurs due to lipotoxicity. Besides, some studies use a combination of mixtures (fructose, sucrose) and fat-rich food components to build metabolic characteristics in rat that affect human characteristics. The purpose of this study was to make an animal model with a metabolic syndrome marker induced by the High Fat Diet (HFD) consisting of pork oil and chicken egg yolk, as well as fructose from simple and economical ingredients. This study was an experimental study using experimental animals of male Rattus norvegicus strain wistar, which were grouped into three random treatment groups, namely the control group, HFD group, and High Fat Diet Fructose (HFDF) group. The number of samples used was 27 rats, with nine rats in each group. The animal was induced for four weeks, then measured levels of FPG, HD, and TG. From the research that has been done, it is found that there are significant differences in levels of Fasting Plasma Glucose (FPG), High-Density Lipoprotein (HDL), and Triglycerides (TG) $(p<0.05)$. It can be concluded egg yolks, lard, and fructose in high-fat diets combined with fructose can increase levels of FPG, TG and reduce levels of HDL, which are markers of the metabolic syndrome used in this study.
\end{abstract}

Keywords: animal models, metabolic syndrome, High Fat Diet, fructose

\section{INTRODUCTION}

Metabolic syndrome (MetS), also known as "syndrome $\mathrm{X}$ " is a metabolic disorder both lipid and non-lipid (Goswami \& Gandhe, 2018), which is defined as a constellation of interrelated physiological, biochemical, clinical, and metabolic factors (Kaur, 2014a). These disorders include insulin resistance, central obesity, hypertriglyceridemia hypertension, and dyslipidemia (Mendrick et al., 2018). Many studies have shown that the prevalence of MetS in extensive ranges from almost $10 \%$ to $84 \%$. The prevalence of MetS is influenced by geographical location, gender, age, race, sedentary lifestyle, high body mass index, and ethnicity (Kaur, 2014a). Furthermore, this is a major public and clinical health challenge throughout the world after urbanization. Metabolic syndrome increases the morbidity and mortality of type 
2 Diabetes Mellitus and cardiovascular disease 2 to 5 times each in the next 5 to 10 years. Individuals with MetS are also susceptible to other conditions of fatty liver, polycystic ovary syndrome, cholesterol gallstones, asthma, sleep disorders, and the appearance of certain types of cancer (Mendrick et al., 2018).

In general, MetS is caused by two factors, namely internal factors, and external factors. Internal (genetic) factors are caused by mutations in genes that encode leptin and its receptors (Ziki \& Mani, 2016), while external factors are influenced by diet, smoking and physical activity (Alarcon et al., 2018). According to the National Cholesterol Education Program Adult Treatment Panel III (NCEP-ATP III) in 2002, the criteria for Asian metabolic syndrome are central obesity (AC >90 cm for men and $>80 \mathrm{~cm}$ for women), High-Density Lipoprotein Cholesterol (HDL-C) levels (men $<40 \mathrm{mg} / \mathrm{dll}$ and women $<50 \mathrm{mg} / \mathrm{dl}$ ), triglyceride levels $>150 \mathrm{mg} / \mathrm{dl}$, blood pressure $>130 / 85 \mathrm{mmHg}$ and fasting blood glucose $>100 \mathrm{mg} / \mathrm{dl}$ (Kaur, 2014b). People with metabolic syndrome can be diagnosed by fulfilling 3 of 5 criteria including obesity and increased waist circumference, increased TG levels, increased blood pressure, hyperglycemia, and decreased serum HDL (Rochlani, Pothineni, \& Kovelamudi, 2017)

Metabolic syndrome is one of the leading causes of death worldwide, and its pathogenic mechanism is not fully understood. The use of animals in experiments is and continues to be very important in medical research, including for the study of metabolism. Animal models or laboratory animals are animals that are treated and made with the aim that it was similar or resembled an object of actual observation as desired. Experimental animal models become important for testing the efficacy of new agents, understanding the molecular basis, pathogenesis, and the mechanism of action of therapeutic agents. However, the results obtained in preclinical studies are not necessarily the same as those found in humans (Fuchs et al., 2018)

High-fat diets will induce insulin resistance in peripheral tissues due to lipotoxicity. Besides, several studies use a combination of carbohydrates (fructose, sucrose) and HFD components to build metabolic syndrome characteristics in rat that imitate human characteristics (Rohman et al., 2017). Excessive consumption of fructose results in an increase in the metabolism of sugar in the liver and causes accumulation of TG and cholesterol due to their lipogenic so that glucose tolerance occurs and causes insulin to become resistant (Wong et al., 2016)

There are several different mouse models for studying MetS, where the focus of making experimental animals can be verified into several learning objectives: 1) models that explain physiopathological and biochemical mechanisms, 2) models in which several MetS signs are induced, and 3) are designed to evaluate therapeutic strategies for overcoming MetS (Barrios-ramos, Loredo-mendoza, \& Jaramillo-flores, 2014).

Giving HFD, which consists of 1 part lard and 1 part chicken yolk eggs for 14 days, can increase triglyceride serum levels. The provision of $100 \mathrm{~g}$ egg yolk and $50 \mathrm{~g}$ lard in white rats can increase cholesterol by $91 \%$ starting on day 14 (Rini, 2012, Hendra et al., 2011). In a study conducted by Rini (2012) evaluating the effect of high-fat diet only on triglyceride levels by comparing the effect of high-fat diet with different types of eggs, namely chicken eggs yolk and duck yolk eggs then research conducted by Hendra (2011) focused on term optimization induction and composition of high-fat diet preparations in female rats, while in this study aims to make animal models in addition to using high-fat diet is also combined with fructose, besides in this study the composition of the high-fat diet used was modified to be simpler than in previous studies. 
This research aims to make an animal model of metabolic syndrome from a simple and easy-to-obtain material, high-fat feed made from chicken egg yolks and pork oil. While the markers of metabolic syndrome to be achieved in this study are increased levels of FPG, decreased levels of HDL and increased levels of TG, that the research focus is on making animal models with the induction of high-fat and fructose feeds by looking at their effects on FPG, HDL, and TG.

\section{MATERIALS AND METHOD}

This study is an experimental research with post-test only control group design, using male white rat (Rattus norvegicus strain wistar) and has approved by Universitas Airlangga Faculty Of Dental Medicine Health Research Ethical Clearance Commission Number: 572/HRECCFODM/VIII/2019, with three treatment groups namely, control group, High Fat Diet (HFD) group, and High Fat Diet + Fructose (HFDF) group. The total number of samples was 27 rats, with nine rat in each group.

This research uses ingredients 1) Standard diet Pokpand with 13-15\% protein composition, $3 \%$ fat, $8 \%$ ash, $6 \%$ fiber, $0.80 \%$ calcium, $0.60 \%$ phosphorus, $13 \%$ moisture content. 2) High Fat Diet (HFD) of $1 \mathrm{ml} / \mathrm{serving}$ consisting of 1 part of pork oil and 1 part of chicken egg yolk with a fat content of $66.28 \%$. 3) liquid fructose with $60 \%$ content.

The experimental animals were acclimatization for seven days, then randomized and divided into three groups, 1) Control group, only gave standard feed 2) HFD group, given standard feed and HFD. 3) HFDF group, given standard feed, HFD, and fructose. Induction is carried out for four weeks. The standard feed is given ad libitum, HFD is given by feeding tube while fructose $60 \%$ is given adlibitum as drinking water.

After induction for four weeks, blood samples were taken from the rat's lateral vein as much as $2 \mathrm{ml}$ (Kumar et al., 2017) and then examined FPG, HDL, and TG with Dimension EXL with LM (clinical chemistry and immunoassay system with $\mathrm{LOCl}^{\circledR}$ chemiluminescence technology) to see the appearance of metabolic syndrome markers. FPG was measured by the hexokinase-UV method using Siemens code DF40 10444971 reagent, HDL was measured by the Liquid Selective Detergent method uses Siemens code DF48B 10464332 reagent and TG were measured by ENZ-Color (GPO) using SIEMEN code DF69A 10444906 reagent.

Statistical data analysis was performed with SPSS 16.0 application. Shapiro-Wilk test was used to determine the normality of data distribution. Afterward, with the Kruskal-Wallis test to assess differences in the average levels of FPG, HDL, and TG between groups, the last Mann-Whitney test was carried out to assess the difference of average levels of FPG, HDL and TG each group.

\section{RESULTS AND DISCUSSION}

Based on the examination result of the FPG, HDL and TG serum levels result are presented on figure 1,2 and 3 . The results of this study indicate differences in the levels of FPG, HDL, and TG in each group after being induced with HFD and HFDF for four weeks.

Figure 1. The results of the study of average levels of FPG after being induced with HFD and HFDF for four weeks showed a difference. The average FPG levels of the control group, HFD, and HFDF were $144.89 \mathrm{mg} / \mathrm{dl}, 173.89 \mathrm{mg} / \mathrm{dl}$, and 328.89 , respectively. 


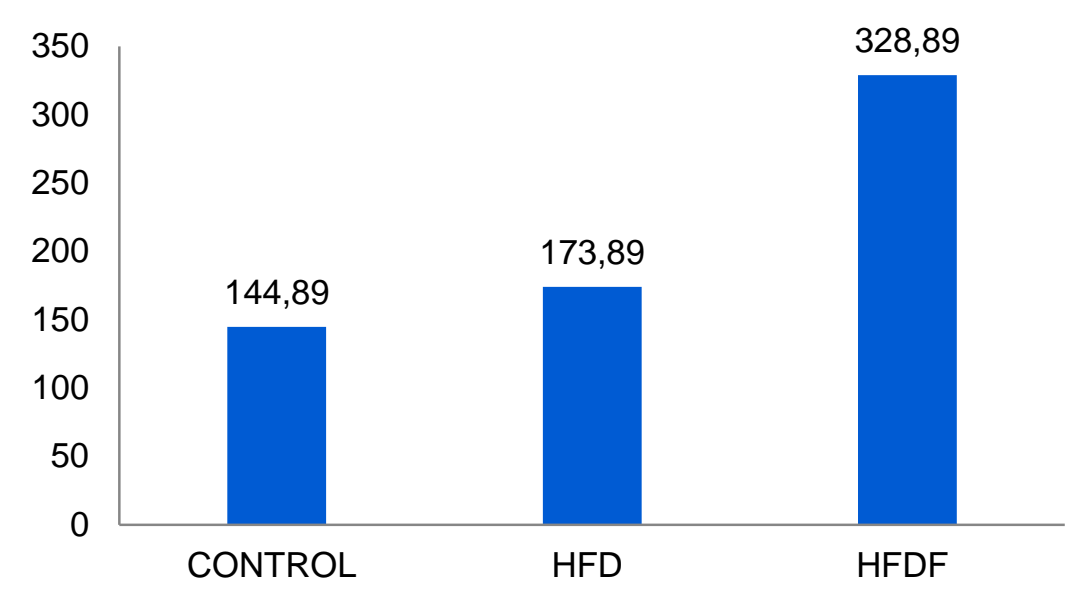

Figure 1 Research results distribution of the average levels of FPG (mg/dl) in the control group, HFD, and HFDF

Figure 2 Results of HDL average levels after induced with HFD and HFDF for four weeks showed a difference. Average HDL levels of the control group, HFD and HFDF were $56.44 \mathrm{mg} / \mathrm{dl}, 40.00 \mathrm{mg} / \mathrm{dl}$, and $33.99 \mathrm{mg} / \mathrm{dl}$, respectively, with the highest HDL levels in the control group.

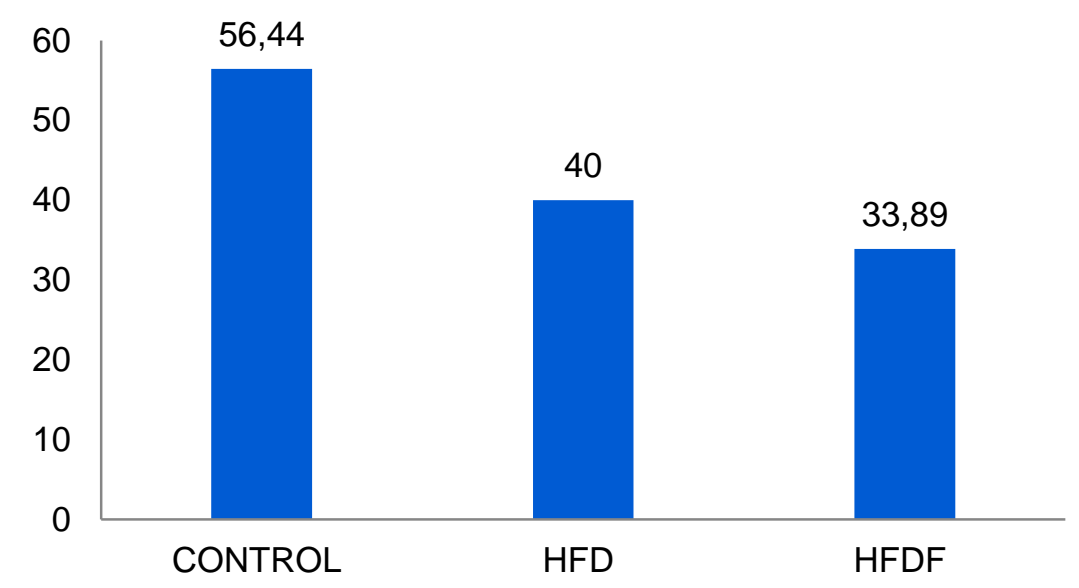

Figure 2. Distribution of research results on average HDL levels $(\mathrm{mg} / \mathrm{dl})$ in the control group, HFD, and HFDF

Figure 3 The results of the study of average TG levels after being induced with HFD and HFDF for four weeks showed a difference. The average levels of TG in the control group, HFD and HFDF were $40.22 \mathrm{mg} / \mathrm{dl}, 77.22 \mathrm{mg} / \mathrm{dl}$, and 122.67 $\mathrm{mg} / \mathrm{dl}$, respectively, with the highest TG levels in the HFDF group. 


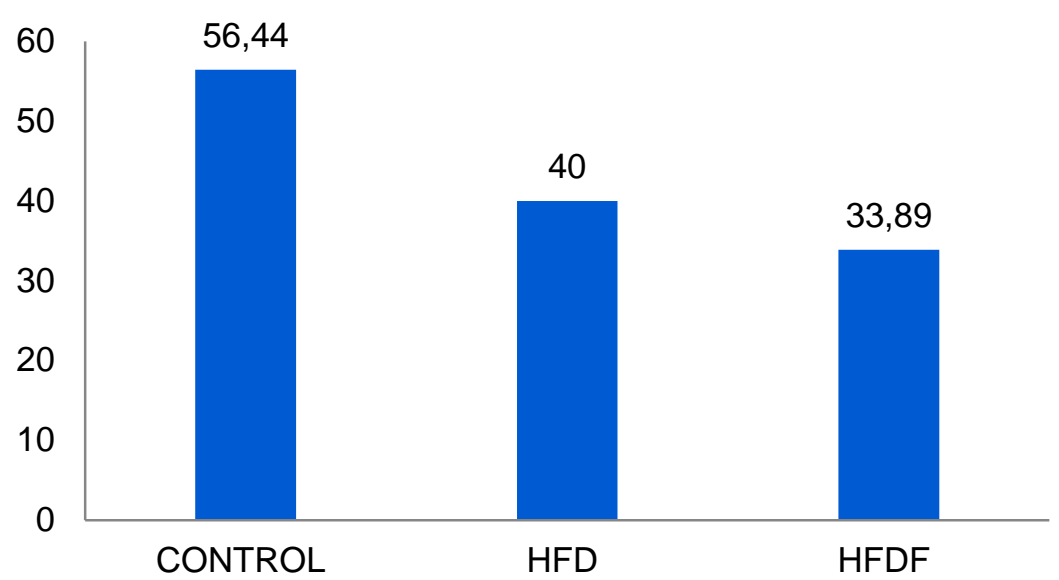

Figure 3. Distribution of research results on average TG levels $(\mathrm{mg} / \mathrm{dl})$ in the control group, HFD, and HFDF

Table 1. Analysis research of research data

\begin{tabular}{ccccll}
\hline Parameter & Control & HFD & HFDF & $\begin{array}{l}\text { P-value } \\
\text { Between } \\
\text {-group }\end{array}$ & $\begin{array}{l}\text { P-value } \\
\text { Each group }\end{array}$ \\
\hline FPG & $144.89 \pm 5.98$ & $173.89 \pm 5.79$ & $328.89 \pm 9.80$ & $0.000^{*}$ & $0.000^{*}$ \\
HDL & $56.44 \pm 1.59$ & $40.00 \pm 2.29$ & $33.89 \pm 1,61$ & $0.000^{*}$ & $0.000^{*}$ \\
TG & $40.22 \pm 2.86$ & $77.22 \pm 7.13$ & $122.67 \pm 9.53$ & $0.000^{*}$ & $0.000^{*}$ \\
\hline
\end{tabular}

${ }^{*} \operatorname{sig} \mathrm{p}<0,05$

Based on the data analysis result, it was found that there were significant differences in each of control groups, HFD and HFDF $(p<0.05)$

From the data presented in Tables 1 and Figures 1, 2, and 3, it can be seen that there are differences in average of FPG, HDL, and TG levels in the control group, HFD group, and HFDF group. Induction of HFD for four weeks affects the increase in FPG and TG levels, and decrease of HDL Level both on HFD and HFDF group compare to the control group.

Research conducted by Marques (2016) also proved that there was a significant increase in blood glucose levels of rats induced by a high-fat diet compared to the group of rats that gave a standard diet. Results in this study are also in line with research conducted by Harsa (2014) that there is an increase in TG levels and a significant decrease of HDL levels in groups of experimental animals induced by the high-fat diet.

Blood glucose levels increased by HFD due to insulin resistance and impaired glucose tolerance (Welty, Alfaddagh, \& Elajami, 2016). The increase in fat consumption is in line with the increase in chylomicron concentration in the blood where the increase in chylomicrons induces insulin resistance through the activation of the Protein kinase $\mathrm{C}$ (PKC) pathway due to high levels of fatty acids which disrupt the activation of the insulin receptor substrate (IRS). Also explained in a previous study, the high free fatty acids will trigger the innate immune system in adipose tissue and then increase the expression of pro-inflammatory adipokines. Free fatty acids can also change the intestinal microbial composition and cause intestinal permeability disorders, thereby increasing lipopolysaccharide activation and 
increasing TNF Receptor Associated Factor 6 (TRAF6). The increase in TRAF6 also contributes to the pro-inflammatory cascade, which activates the NF-kB signaling. Thus, a high-fat diet can trigger glucose tolerance through insulin resistance (Harsa, 2014; Rohman et al., 2017).

Based on the analysis presented in table 1, it can be seen that there are significant differences between FPG, HDL, and TG levels in the HFD group compared to the HFDF group. These data indicate that the increase of FPG levels in the HFDF group was higher than in the HFD group. Also, an increase in TG levels was higher in the HFDF group compared to the HFD group, whereas in HDL levels, there was a significant decrease in levels in the HFDF group compared to the HFD group.

Result in this study also in line with research conducted by MorenoFernandez (2018) and Barios-Ramos (2014) both studies showed that the effect combining fructose and feed in increasing levels of FPG and TG level in the group of experimental animals that were inducted with fructose. Fructose consumption increases TG levels and decreases HDL-C levels. Several animal studies have shown that high-fructose diets are associated with hypertriglyceridemia, impaired glucose tolerance, hyperinsulinemia, insulin resistance, and increased blood pressure, and weight gain (Kelishadi, Mansourian, \& Heidari-beni, 2014). An increase in TG and cholesterol levels is associated with the absorption of fructose into the liver in high amounts due to lipogenic properties, which then lead to insulin resistance and glucose tolerance (Tappy, Le, Tran, \& Paquot, 2010).

Fructose in the liver by the presence of phosphofructokinase and ATP enzymes that cause catalysis reactions resulting in the conversion of fructose to fructose-1-phosphate (Aydin et al., 2014). Then enter the initial stages of glycolysis by the formation of glyceraldehyde and dihydroxyacetone without passing through fructose-1,6-bisphosphate. Fructose can continue through the glycolysis pathway by the negative regulator Phosphofructokinase so that it requires several processes to convert pyruvate to lactate, combine phosphate through the necessary glucogenesis, fructose used to become fat, fructose used by lipid oxidation which must be converted by carbohydrate to be metabolized more frequently too high for pyruvate, lactate is needed, glycerol, and acyl-glycerol molecules. (Wong et al., 2016)

Dyslipidemia is a result of which occurs when fat levels in the bloodstream are too high or too low. Triglycerides and cholesterol that are synthesized by the liver hydrolysis in circulation by lipoprotein lipase as well chylomicron enghidrolisis into smaller lipoprotein particles. LDL is the lipoprotein that contains the most cholesterol $(60-70 \%)$. The amount of cholesterol that will be oxidized depends on the cholesterol level contained in LDL. Some conditions affect the level of oxidation, such as increasing the amount of LDL as in metabolic syndrome and HDL cholesterol levels, the higher the HDL level, the HDL is protective against LDL oxidation (Rochlani, Pothineni, and Kovelamudi 2017).

FFA is the main source of TG synthesis in the liver which results in the stability of lipoprotein production where increased lipoprotein particles will contribute to increasing VLDL (Nurul et al. 2019). Insulin, which replaces lipolysis, becomes FFA. Insulin in the PI3K pathway also dramatically influences the increase in VLDL. Insulin is performed as a mediator of VLDL circulation through lipase activity. Of these three things, insulin resistance becomes closely related to VLDL and hyper-TG (Gustavo et al. 2017). Thus, an increase in TG concentration in insulin resistance results from an increase in VLDL and a reduction in VLDL elimination (Lanktree and Hegele 2017). HDL which is quickly withdrawn from the circulatory system, which 
reduces the transport of cholesterol in the blood (Pirillo and Catapano 2013). In individuals with insulin resistance, there is an increase in FFA circulation, TG synthesis, and storage is increased, then this TG excess is synthesized into VLDL VLDL. Dyslipidemia which correlates with insulin resistance due to increased VLDL secretion in the liver. (Dashty, 2014; Esfahani, Baranchi, and Taghi 2019).

According to NCEP-ATP III, metabolic syndrome has five primary markers, however, with the fulfillment of three markers, it can be declared metabolic syndrome, but further research is needed in order to meet the criteria of the best animal metabolic syndrome model that fulfills the five criteria. In addition to providing high fat and fructose diet in the long term vulnerable to cause animals to become stressed, it is important to carry out further research on a short and effective period to get an animal model of metabolic syndrome so that it is not susceptible to stress.

\section{CONCLUSION}

Egg yolks, lard, and fructose in high-fat diets combined with fructose can increase levels of FPG, TG, and reduce levels of HDL, which are markers of the metabolic syndrome used in this study.

\section{ACKNOWLEDGMENT}

The author, would like to thank those who have helped to carry out this research, especially in the immunology study program faculty of postgraduate school Airlangga University, Laboratory of Biochemistry Faculty of Medicine, Airlangga Unversity and Clinical Pathology Laboratory Dr. Soetomo Surabaya who have facilitated this research.

\section{REFERENCE}

Alarcon, G., Roco, J., Medina, M., Medina, A., Peral, M., \& Jerez, S. (2018). High fat diet-induced metabolically obese and normal weight rabbit model shows early vascular dysfunction: mechanisms involved. International Journal of Obesity. https://doi.org/10.1038/s41366-018-0020-6

Aydin, S., Aksoy, A., Aydin, S., Kalayaci, M., Yilmaz, M., Kuloglu, T., ... Catak, Z. (2014). Today ' s and yesterday' s of pathophysiology: Biochemistry of metabolic syndrome and animal models. Nutrition, 30(1), 1-9. https://doi.org/10.1016/j.nut.2013.05.013

Barrios-Ramos, Loredo-mendoza, \& Jaramillo-flores. (2014). A Quick Model for the Induction of Metabolic Syndrome Markers in Rats, 4(1), 1-5. https://doi.org/10.4172/2165-8048.1000137

Dashty, M. (2014). Diabetes \& Metabolism A Quick Look at Biochemistry: Lipid Metabolism, 5(1), 1-19. https://doi.org/10.4172/2155-6156.1000324

Fuchs, T., Loureiro, M. de P., Macedo, lano eMerson, Nocca, D., Nadelcu, M., \& Costa-casagrande, T. andrade. (2018). Animal models in metabolic syndrome., 45(5), 1-10. https://doi.org/10.1590/0100-6991e-20181975

Goswami, K., \& Gandhe, M. (2018). Evolution of Metabolic Syndrome and its Biomarkers. Diabetes \& Metabolic Syndrome: Clinical Research \& Reviews. https://doi.org/10.1016/j.dsx.2018.06.027

Harsa, I. M. S. (2014). Efek Pemberian Diet Tinggi Lemak Terhadap Profil lemak Darah Tikus Putih (Rattus norvegicus). Jurnal IImiah Kedokteran, 3(1), 21-28.

Hendra, P., Wijoyo, Y. F., \& Dwiastuti, R. (2010). Optimasi Lama Pemberian dan Komposisi Formulasi Sediaan Diet Tinggi Lemak Pada Tikus Betina. Laporan Penelitian. 
Kaur, J. (2014a). A Comprehensive Review on Metabolic Syndrome, 2014. https://doi.org/10.1155/2014/943162

Kaur, J. (2014b). Assessment and Screening of the Risk Factors in Metabolic Syndrome, 140-152. https://doi.org/10.3390/medsci2030140

Kelishadi, R., Mansourian, M., \& Heidari-beni, M. (2014). Association of fructose consumption and components of metabolic syndrome in human studies: A systematic review and meta-analysis. Nutrition, 30(5), 503-510. https://doi.org/10.1016/j.nut.2013.08.014

Kumar, M., Dandapat, S., Sinha, M. P., \& Kumar, A. (2017). Different blood collection methods from rats: A review. Balneo Research Journal, 8(1), 46-50. https://doi.org/DOI: http://dx.doi.org/10.12680/balneo.2017.141

Mendrick, D. L., Diehl, A. M., Topor, L. S., Dietert, R. R., Will, Y., Merrill, M. A. La, ... Burleson, F. G. (2018). Metabolic Syndrome and Associated Diseases: From the Bench to the Clinic. Toxicological Science, (January), 1-7. https://doi.org/10.1093/toxsci/kfx233

Rini, S. (2012). Pengaruh pemberian diet tinggi lemak terhadap kadar trigliserida pada tikus (Doctoral dissertation, Universitas Muhammadiyah Surakarta).

Rochlani, Y., Pothineni, N. V., \& Kovelamudi, S. (2017). Metabolic syndrome: pathophysiology , management , and modulation by natural compounds, 215225. https://doi.org/10.1177/https

Rohman, M. S., Lukitasari, M., Nugroho, D. A., \& Widodo, N. (2017). Development of an Experimental Model of Metabolic Syndrome in Sprague Dawley Rat. Research Journal of Life Science, 04(01), 76-86.

Tappy, L., Le, K. A., Tran, C., \& Paquot, N. (2010). Fructose and metabolic diseases: New findings, new questions. Nutrition, 26(11-12), 1044-1049. https://doi.org/10.1016/j.nut.2010.02.014

Welty, F. K., Alfaddagh, A., \& Elajami, T. K. (2016). Targeting inflammation in metabolic syndrome. Translational Research, 167(1), 257-280. https://doi.org/10.1016/j.trsl.2015.06.017

Wong, S. K., Chin, K., Suhaimi, F. H., Fairus, A., \& Ima-nirwana, S. (2016). Animal models of metabolic syndrome: a review. Nutrition \& Metabolism, 1-12. https://doi.org/10.1186/s12986-016-0123-9

Ziki, M. D. A., \& Mani, A. (2016). Metabolic syndrome : genetic insights into disease pathogenesis, 27(2), 162-171. https://doi.org/10.1097/MOL.0000000000000276 